\title{
Report
}

\section{Using Physical Factor Therapy for Postoperative Patients with Cerebral Apoplexy Voiding Dysfunction: A Case Study}

\author{
Du Fei ${ }^{1}$, Li Tong ${ }^{1}$, Wang Chengshuang ${ }^{2}$, Wang Lin ${ }^{2}$, Kong Fan ${ }^{3}$, Zheng Wanjun ${ }^{1}$, Xu Xue ${ }^{1}$, \\ Cheng Hongwei ${ }^{1}$, Liang Chengjun ${ }^{2,}$, , Yan Zhi ${ }^{1,2, *}$ \\ ${ }^{1}$ Department of Rehabilitation, Dalian Port Hospital, Dalian, China \\ ${ }^{2}$ College of Physical Education, Liaoning Normal University, Dalian, China \\ ${ }^{3}$ School of Kinesiology and Promotion, Dalian University of Technology, Dalian, China
}

\section{Email address:}

dufei198813@126.com (Du Fei), Hys907325@163.com (Li Tong),1971370680@qq.com (Wang Chengshuang),

2364314531@qq.com (Wang Lin),2438383590@qq.com (Kong Fan),382574612@qq.com (Zheng Wanjun),376167394@qq.com (Xu Xue), 253465575@qq.com (Cheng Hongwei), liangchengjun126@126.com (Liang Chengjun), qjqiaojie@qq.com (Yan Zhi)

${ }^{*}$ Corresponding author

\section{To cite this article:}

Du Fei, Li Tong, Wang Chengshuang, Wang Lin, Kong Fan, Zheng Wanjun, Xu Xue, Cheng Hongwei, Liang Chengjun, Yan Zhi. Using Physical Factor Therapy for Postoperative Patients with Cerebral Apoplexy Voiding Dysfunction: A Case Study. Rehabilitation Science. Vol. 6, No. 2, 2021, pp. 20-24. doi: 10.11648/j.rs.20210602.11

Received: April 2, 2021; Accepted: April 15, 2021; Published: April 29, 2021

\begin{abstract}
Objective: We introduce and analyze by physical factors treatment case the method and effect of urinary retention after stroke, li xx hospital diagnosis: patients with cerebral hemorrhage postoperative recovery, on the left side of the limb dysfunction, urinary bladder sensitivity is poorer, lack of detrusor activity, urethral sphincter muscle tension increased, urination disorders, urinary retention. Treatment methods: Using electrical therapeutic apparatus, electrodes cross placed in the lower abdomen in patients with bladder section. Using percutaneous nerve electrical stimulation, placed therapeutic apparatus is the cathode respectively in patients with the third sacral vertebral and bladder reflex zone, set the treatment frequency is 120 $\mathrm{hz}$, voltage range of $20 \sim 26 \mathrm{v}$. Using shortwave treatment, set the short-wave frequency therapy apparatus $10 \mathrm{w}$, before and after the placement of electrodes, to help urethral sphincter muscle tension relaxed, eliminate urinary tract inflammation and edema. Results: By interfering with the treatment, percutaneous neuromuscular electrical stimulation (TENS) and short wave 15 days after treatment, patients can feel obviously can urinate and pa ontaneously $300 \mathrm{ml}$ urine volume. Conclusion: interference electric treatment can stimulate the detrusor contraction, short-wave can improve urethral sphincter muscle tension, Percutaneous neuromuscular electrical stimulation therapy can excitement parasympathetic promote bladder function, improve urinary retention in patients with quality of life.
\end{abstract}

Keywords: Cerebral Apoplexy, Urinary Retention, Interfere with the Electrical Treatment, Percutaneous Nerve Electrical Stimulation Therapy, Shortwave Treatment

\section{The Introduction}

Also called cerebrovascular accident or stroke, stroke is refers to the various causes of cerebral vascular rupture or blocked a group of disease, parenchymal damage can be divided into the ischemic stroke and ischemic stroke. According to one report, the incidence of stroke in our country is still increasing year by year, because of the death toll to 1.57 million people in 2018 , became the malignant tumor and the third largest national cause of death after heart disease [1]. Stroke will usually cause many complications, urinary retention is one of common complications [2]. Studies have found that stroke after the injured brain tissue may lead to brain micturition center or micturition signal conduction nerve by different degrees of damage, cause detrusor dysfunction, micturition related muscle coordination disorder [3], bladder sensory dysfunction in patients with urinary retention $[4,5]$. A study shows that $50 \%$ of the 
patients with cerebral apoplexy occur urinary retention, there are $15-20 \%$ of patients 6 months after surgery urinary retention problems still [6]. The diagnosis of urinary retention is not difficult, the clinical symptoms are usually characterized by abdominal distension, pain and dysuria. But need to determine the specific reasons for further analysis of the urine dynamics research. According to a study in ischemic stroke patients and in patients with ischemic stroke, $70.7 \%$ of patients will appear in the former detrusor excessive activity, $29.3 \%$ detrusor activity is insufficient, the latter in $34.6 \%$ of patients will appear detrusor excessive activity, $65.4 \%$ detrusor activity is insufficient. The blood type than ischemic stroke are more likely to appear detrusor contractions are unable to [7], the patients with dysuria. Need timely treatment, and clinically in urinary retention, otherwise it will cause the expansion of the urinary tract, urinary tract infection and kidney disease patients' life. Clinical indwelling catheterization and intermittent catheterization is the most common method to treat urinary retention, but a prolonged treatment may cause urinary tract infection or urinary tract injury [8]. And pelvic floor muscle training, bladder function training effective physical therapy methods, such as slow and patient compliance is poor, can't meet the demand of patients with urination as soon as possible. Electrical stimulation therapy has no traumatic and the characteristics of easy operation, and a large number of domestic and foreign literature shows that electrical stimulation can create excitement parasympathetic quickly lower central micturition reflex mechanism; Stimulate the detrusor contractions, urinate urethral sphincter coordination movement; Stimulate bladder afferent mechanoreceptor improve bladder sensory organs, so as to restore micturition function [9-12], for the treatment of urinary retention after a stroke has great value. Clinical common electrical stimulation treatment including interference electricity, percutaneous neuromuscular electrical stimulation therapy. In addition, the short wave therapy is often used to improve the urethral sphincter muscle tension and eliminate the local inflammation and edema caused by indwelling catheter.

\section{Case Descry}

Li xx, female, 69 years, left limb dysfunction in patients with 24 days, October 14, 2020 in our hospital rehabilitation treatment. Chief complaint: on the left side of the limited physical activity, water choke to cough, difficulty swallowing, no urine, dysuria, urinary retention. Auxiliary examination, CT examination showed low density shadow: right frontal, parietal and temporal bone, pillow acute subdural hematoma produce cerebral hernia; Urine dynamics test shows: the detrusor contraction ability to reduce shrinkage to maintain a shorter time, higher external urethral sphincter muscle tension, detrusor contractions, around the urethra to involuntary striated muscle contraction, bladder residual urine volume. Now legacy left limbs activity limitations, and swallowing function decline in drinking water, the life cannot provide for oneself, dizziness, headache, nausea, vomiting, chest pain and other symptoms, nasogastric diet, urinary tube. Medical history: a history of type 2 diabetes, hypertension, oral antihypertensive drug erratic, unclear blood pressure control. Specialized examination: conscious, not words, listening comprehension, cognitive decline, on the left side of the body movement dysfunction, urinary bladder reduced susceptibility, difficult urine, urinary retention. Diagnosis: cerebral hemorrhage postoperative recovery time, on the left side of the limb dysfunction, urinary bladder sensitivity is poorer, lack of detrusor activity, urethral sphincter muscle tension increased, urination disorders, urinary retention.

\section{Methods and Results}

Patients with voiding dysfunction, need, placing a urinary catheter urethra, easy injury lead to urinary tract infections, seriously affected the patients' body movement function, daily life ability, unnecessary trauma and pain. Therefore, decided to give patients, director of the department of physiotherapy li tong treatment of physical factors: increasing detrusor muscle strength and sensitivity to interfere with the electrical treatment; Micturition center TENS treatment; Shortwave treatment improve urination muscle tension.

\subsection{Interference Electric Treatment}

Interference power is a kind of low frequency modulated medium frequency current, body can form interference electric current in the patients. Bladder rely mainly on detrusor sphincter and to control the storage of urine and urine [13], rhythmic interference electric current to stimulate your pelvic floor muscles, acting on the bladder, improve its shrinkage ability, increase the internal pressure, adjusting control sphincter and detrusor nerve reflex [14], promote the bladder muscles rhythmic movement, ease the urethral sphincter muscle spasm, so as to enhance the capacity of automatic micturition in patients with [15]. Operation method is: set up interference electric therapeutic instrument carrier frequency of $4 \mathrm{KHZ}$, frequency conversion output of $30 \sim 50$ $\mathrm{hz}$ (IFC model 15) therapeutic electrodes cross placed in the lower abdomen in patients with bladder area, $20 \mathrm{~min} / \mathrm{times}$ and 6 times per week.

\subsection{Micturition Center TENS Therapy}

Percutaneous neuromuscular electrical stimulation therapy is one of the clinical widely used to treat urinary retention method [16], third placed therapeutic apparatus is the cathode respectively in patients with sacral vertebral and bladder reflex zones, treatment frequency is $120 \mathrm{hz}$, the voltage range of $20 \sim 26 \mathrm{v}$ (tolerance advisable, with patients with urine in patients with muscle increased sensitivity can gradually reduce the current), $20 \mathrm{~min} /$ times and 6 times per week. Application of therapeutic plus electrical stimulation to adjust to improve and restore the body disorder of biological 
electromagnetic fields, promote blood circulation of pelvic floor muscles, activate the neuromuscular system, thus stimulating the contraction of the pelvic floor muscles.

\subsection{Shortwave Treatment}

Operation method: as the older patients, treatment dose should be appropriate. Set shortwave therapeutic dose of 10 $\mathrm{w}$, opposed before and after placement of electrodes (one electrode in patients with lower abdominal, another electrode placed in the patients with sacral sacral $2 \sim 4$ ), $15 \mathrm{~min} /$ times, six times per week, shortwave treatment of sympathetic nerve and plant nerve excitability two-way adjustment, stimulate the pelvic floor muscle layer depth, improve the bladder detrusor excitability, improve sphincter muscle tension, relieve spasm, promote urination function recovery [17], in addition, long-term indwelling catheter, easy cause urinary tract inflammation, short-wave therapy is helpful to reduce inflammation and swelling.

\subsection{The Results}

After 2 weeks after treatment, patients with bladder sensitivity significantly enhance, improve sphincter muscle tension, detrusor systolic blood pressure significantly reduced, feel have meaning is, pull out the ureter, urinary tract unobstructed, urine flow rate increased, urine discharge from $300 \mathrm{ml}$, intermittent catheterization success, B ultrasonic examination was performed after micturition, results showed that bladder residual urine volume less than $50 \mathrm{ml}$, urinary retention improvement.

\section{Discussion}

\subsection{The Causes of Urinary Retention and Conventional Treatments}

Urinary retention is the classification of urinary retention due to bladder filling too much urine but unable to rule out or does not appear too much residual urine effective emptying. From the onset time can be divided into acute urinary retention and chronic urinary retention, middle-aged and old women is common causes of urinary retention in the bladder, urethra itself or organic around the lesions caused by mechanical obstruction, in spasms, stones, tumor, tuberculosis, bladder neck fibrosis, after pelvic radiotherapy, another common diseases because of dynamic obstruction, is caused because of neuromodulation dysfunction, seen in trauma, chronic disease (diabetes, high blood pressure), medication (Plutarch benzene sheen, 654-2, atropine and other), hypokalemia, and coma. Individual patients is due to not accustomed to bed urination.

\subsection{Diagnosis}

First ask, attaches great importance to the history of chronic urinary retention patients often have urinary tract symptoms, such as frequency, urgency, urine is not net, urinary incontinence, etc., combined chronic diseases, such as sugar urine disease, high blood pressure, respiratory system disease.

\subsection{Stroke Urinary Retention}

As the main clinical type of cerebrovascular disease, stroke, also known as stroke, including ischemic stroke and hemorrhagic stroke, suddenly fainted, unconscious, companion comes, bad language, such as hemiplegia for clinical primary symptom of disease. Is currently the third leading cause of death in our country. Most persons over the age of 65 patients with cerebral apoplexy, has a high incidence, the characteristics of the high morbidity and high mortality. And stroke can cause a series of disorders, such as cognitive dysfunction, excretion dysfunction, speech dysfunction, urinary retention is a common complication after stroke, clinical manifestations mainly is unable to urinate or dysuria [18], and related factors on urinary retention after stroke in recent years has been widely discussed. Researchers think of urinary retention after stroke and cognitive dysfunction, speech dysfunction and so on, but has not be confirmed. In recent years, studies have found that speech dysfunction patients are more likely to be because of language barrier and it's hard to show their urine production, demand and some patients with more severe cognitive dysfunction may even be aware of these problems. And domestic research shows that the occurrence of urinary retention after stroke associated with age, sex, has the certain $[19,20]$. In addition, the researchers think feel urinary retention and urinary bladder damage after stroke and to reduce the detrusor contraction force, but there are also studies have shown that reflex bladder appeared during the period of acute stroke patients in the subsequent urine dynamics test results is normal, suggest that abnormal detrusor activity is likely to be short-lived, so the detrusor dysfunction may have nothing to do with urinary retention $[21,22]$.

\subsection{The Treatment of Urinary Retention}

At present about the treatment of urinary retention after stroke mainly includes surgical treatment, drug therapy, indwelling urethral catheterization, intermittent catheterization, physical therapy and Chinese medicine treatment, etc. [23]. Indwelling catheter is clinical commonly used treatments, but long-term indwelling catheter bacteria, patients with urinary system infection. At the same time, long-term indwelling catheterization can easily cause micturition muscle dysfunction, micturition function of patients with lead to further decline. Then the researchers introduced the intermittent catheterization, and research has shown that intermittent catheterization relative to indwelling urethral catheterization, in the lower urinary tract infection and the risk of micturition muscle dysfunction at the same time, can promote the recovery of micturition muscle function in patients with, increase urine output, less urine in bladder allowance [24]. On drug control, mainly including two aspects of traditional Chinese medicine and western medicine. Chinese medicine is 
mainly some diuresis, nerves of traditional Chinese medicine. Shu-hong li [25] such asthrough the kidney bolus plus five golden three intervention in 40 patients with urinary retention, it is found that in addition to the seven patients need indwelling catheter, the rest of the patients have different degree of curative effect, shows that Chinese medicine intervention in the treatment of urinary retention has played a certain effect. In western medicine mainly cholinergic preparation and injection of prostaglandin in bladder, Brian S Buckley, [26] by collecting drugs for the treatment of urinary retention literature found that the injection of prostaglandin and success in bladder urination found statistically significant correlation between, compared with placebo, emptying cholinergic drugs combined depressant and nature there is a statistically significant correlation between the improvement in possibility. In addition to drug therapy and urethral catheterization for patients adjuvant therapy has become a hot research topic. Zhong-hua wang etc. [27] through the use of electric acupuncture with bladder function training to improve voiding dysfunction of patients with urinary retention after stroke, it was found that patients with electric acupuncture intervention with bladder function training, 41 patients were cured, 13 cases were improved, effective for 8 cases of patients, including instructions with bladder functional training can promote patients to urinate, reduce residual urine in bladder, can be used as a clinical treatments for promotion of urinary retention. Although now about the treatment of urinary retention after stroke means there are many, both at home and abroad for surgical treatment, drug therapy, physical factor, in the form of traditional Chinese medicine and the research; But so far, there isn't a way for the treatment of urinary retention recognized [28].

\subsection{This Case the Treatment of Urinary Retention}

This research adopts the interference treatment, micturition center TENS therapy, short-wave therapy three physical factors in patients with urinary retention after stroke treatment, treatment of physical factors relative to other treatments, a noninvasive, convenient operation, wide applicable people, less cost, in patients with urinary retention after stroke has great popularity. Treatment of physical factors using electricity, light, sound, in the role of magnetic physical factors such as the human body, through the physiological regulation mechanism of nerve or endocrine system to achieve treatment [29]. This research use electrical stimulation treatment method is mainly used to reconstruct the lower central micturition reflex, stimulate the detrusor contractions, increased urination function in patients with sensory input mechanism to promote recovery. Results found that patients in began on December 15, 2020 for treatment of physical factors and treatment for half a month later, on December 30, 2020 12:00 on urination, urine volume $300 \mathrm{ml}, 12: 10$ orders intermittent catheterization time, $30 \mathrm{ml}$ of urine, 18:00 orders intermittent catheterization time, $30 \mathrm{ml}$ of urine, relative to accept physical factors increase automatic micturition before treatment. And urine flow dynamic test was performed, and found that patients with bladder residual urine relative physical factors reduce before treatment. Therefore, the way of physical therapy intervention for patients with urinary retention after stroke has certain therapeutic effect, so the future can add physical factor in patients with more such treatment, improve curative effect.

\section{Conclusion}

Urinary retention can cause urinary tract infections, kidney seeper complications such as, should be early found early treatment. For the patients with urinary retention after stroke, postoperative residual limb dysfunction reduce patient adherence, bladder function training treatment cannot achieve the treatment effect, and treatment of physical factors of the advantages of simple operation, no wound, curative effect is distinct, is worth us to continue to further explore the grope, play a role of the clinical better, better service for urinary retention after stroke patients.

\section{Acknowledgements}

This work was supported by the Natural Science Foundation Guidance Project of Liaoning Province, China (2019-ZD-1000, 2019-ZD-1002).

\section{References}

[1] Yong-jun wang, Li Zixiao GuHongQiu etc. Chinese stroke report 2019 (in Chinese) (1) [J]. Chinese journal of stroke, 2020, (10): 1037-1043.

[2] Umemura $\mathrm{T}$, Ohta $\mathrm{H}$, Yokota $\mathrm{A}$, et al. The Urinary retention associated with stroke [J]. Journal of UOEH, 2016, 38 (4): 263-269.

[3] Chou Y C, Jiang Y H, Harnod T, et al. The Stroke and the lower urinary tract symptoms: A neurosurgical view [J]. Journal of Urological Science, 2019, 30 (1): 8.

[4] fionna, nitti vw, Liu Liyan, etc. The bladder lumen electrical stimulation of neurogenic bladder sensory function [J]. The influence of the theory and practice of Chinese rehabilitation, 2010, 16 (12): 1106-1107.

[5] Fang Qiang, song bo. The formation mechanism of bladder sensory function and detection method research progress $[\mathrm{J}]$. Journal of clinical urology, 2006, 8 (21): 638-640.

[6] Naganuma M, Inatomi Y, Yonehara $\mathrm{T}$, et al. The Urinary retention associated with unilateral medullary infarction $[\mathrm{J}]$. J Rinsho shinkeigaku $=$ Clinical neurology, 2005, 45 (6): 431-436.

[7] Han K S, Heo S H, Lee S J, et al. Comparison of urodynamics between ischemic and hemorrhagic stroke patients; Can we suggest the category of urinary dysfunction in patients with cerebrovascular accident "according to the type of stroke? [J]. Journal of Neurourology and Urodynamics: Official Journal of the International Continence Society, 2010, 29 (3): 387-390. 
[8] PuJing, Chen yonghong, He Lahui. The value of high quality nursing management in controlling hospital infection [J]. Journal of modern medicine, 2016, 22 (36): 134-135.

[9] Ersoz M, Tunc H, Akyuz M, et al. The Bladder storage and emptying disorder frequencies in hemorrhagic and ischemic stroke patients with Bladder dysfunction. Cerebrovasc Dis 2005; 20: 395 to 9 .

[10] Liu Yi Xu Gang, Huang Jing. Different frequency of percutaneous nerve electrical stimulation to improve the comparison of urinary incontinence after stroke [J]. China rehabilitation theory and practice, 2015, 21 (5): 562-566.

[11] Monteiro, ES, De Carvalho LB, Fukujima MM, et al. Electricalstimulation of the posterior tibialis nerve little symptoms of poststrokeneurogenic overactive bladder in men: a randomized controlled trial [J]. Journal of Urology, 2014, 84 (3): $509-514$.

[12] Liao Zhiying. Interfere with electrical stimulation treatment on urinary incontinence in patients with stroke symptoms $[\mathrm{J}]$. The influence of contemporary Chinese medicine, 2018, 025 (031): 76-79.

[13] Yang Quanxing Zeng Li, BiShiQing etc. Interference electric and intermediate frequency electric observation of effects of urination function after surgery for cervical cancer [J]. Journal of zunyi medical college, 2010 (6): 554-555.

[14] To wei neosaurus. Acupuncture combined interference treatment the clinical effect of the treatment of urinary retention after stroke $[\mathrm{J}]$. Journal of Inner Mongolia traditional Chinese medicine, 2020, 33 (3) 6: 116-117.

[15] hong-xia liu. Clinical observation on integrated treatment of acute urinary retention after stroke $[\mathrm{J}]$. Journal of practical medicine magazine, 2015, 31 (6): 558.

[16] Peak. WeiZhe, cheng from TNES therapeutic research and clinical application $[\mathrm{J}]$. Journal of northwest national defense medical journal, 1993 (02): 53.

[17] Ma Shuyi ZhenYuTing. With the effect of ultrashort wave therapy of diabetic neurogenic bladder study [J]. Journal of contemporary medical sciences, 2017, 15 (07): 40-41.
[18] xue-lian Yang, Li Longxuan. The research progress of urinary retention after stroke [J]. World of clinical medicine, 2019, 40 (10): 671-674.

[19] Zhang Deyong. After acute cerebral apoplexy urination disorder related factors analysis [J]. Chinese journal of practical neurological diseases, 2014 (10): 57-59.

[20] Wang. After acute cerebral apoplexy urination disorder related factors analysis $[\mathrm{J}]$. Journal of Chinese medicine science, 2011. 1 (16): 174-175.

[21] LuHaiTao Zhang Tong. Recovery of urinary dysfunction related to stroke, predictor and urine flow mechanics $[\mathrm{J}]$. Chinese neuroscience journal, 2010 (01): 56-59.

[22] Kim, T. G., etal. The Outcomes of drug - resistant urinary retention in patients in the early stage of stroke [J]. Ann Rehabil Med, 2015, 39 (2): p. 262.

[23] Zhang Keming, Li Jinfang. Treatment of bladder dysfunction after central nervous injury [J]. International journal of neurology, neurosurgery, 2015, 42 (6): 556-560.

[24] Huang Houqiang etc. Intermittent catheterization effect on urinary retention in patients with cerebral apoplexy [J]. Journal of practical medical journal, 2018, 34 (03): 482-486.

[25] Li Shuhong, Li Zhanmin, Zhang Li. The needle medicine treatment urination disorder after stroke 40 cases [J]. Journal of liaoning traditional Chinese medicine journal, 2006, 33 (5): $579-579$.

[26] Buckley, B. S. and McCain Lapitan, Drugs for treatment of urinary retention after surgery in adults $[\mathrm{J}]$. The Cochrane Database price Rev, 2010 (10): p. CD008023.

[27] zhong-hua wang and Yang Bo. Electric acupuncture treatment of urinary retention after stroke with bladder function training of clinical observation [J]. Modern journal of urology, 2009. 14 (02): 121-122.

[28] Han Shuxia, Xie Ying. Treatment of physical factors in the application of neurogenic bladder [J]. China rehabilitation theory and practice, 2015, 21 (12): 1430-1432. 